\title{
Effects of Tool Coupling on Transient Simulation of a Mobile Air-Conditioning Cycle
}

\author{
Roland Kossel $^{1} \quad$ Nils Christian Strupp ${ }^{2} \quad$ Wilhelm Tegethoff $^{1}$ \\ ${ }^{1}$ TLK-Thermo GmbH \\ ${ }^{2}$ TU Braunschweig, Institut für Thermodynamik \\ Hans-Sommer-Str. 5, 38106 Braunschweig \\ r.kossel@tlk-thermo.de
}

\begin{abstract}
Results of numerical simulations more and more provide a basis for design decisions in an automotive context. When simulating complex systems, one of two approaches can be chosen: The modeling in one multidomain language like Modelica or the utilization of different specialized simulation programs.

This paper demonstrates the simulation of the Heating Ventilation and Air-Conditioning system (HVAC) of a car. The different components are modeled individually and validated with measurement data in separate test benches. A co-simulation using one Dymola instance per component model is then created to represent the whole refrigeration cycle taking into account the inter-component dependencies.

To evaluate the effects introduced by the tool coupling, the results are compared to those of a single Modelica model composed of all component models.
\end{abstract}

Keywords: tool coupling; co-simulation; refrigeration

\section{Introduction}

Results of numerical simulations more and more provide a basis for design decisions in an automotive context. This also applies for the thermodynamic subsystems for example the Heating Ventilation and AirConditioning system (HVAC).

This paper discusses dynamic simulations of an automotive refrigeration cycle with Modelica using the TIL library and Dymola. A R134a cycle with detailed components is used. Each component model is validated separately using measurement data from a broad range of ambient conditions.

There are two approaches for modeling and simulating complex systems composed of multiple compo- nents: Use a suitable language to describe the complete system in one model or divide the system into submodels, then employ different simulation programs specialized for the respective subproblems and use cosimulation to create a model of the complete system.

The required level of detail plays an important role in the decision for either approach. If for example a simulation of the HVAC unit and the passenger's compartment of a car shall be conducted, the models could be created using just Modelica. If however the goal of the whole simulation is an evaluation of the temperature distribution within the compartment, a $3 \mathrm{~d}$ simulation tool must be used; because the HVAC unit can be represented only poorly by $3 \mathrm{~d}$ tools, a co-simulation makes sense [5].

Under specific circumstances it is even practical to create a co-simulation with multiple instances of one tool. The decision must be made considering two main points: The simulation speed and the numerical stability of the simulation. For both points no general rule can be given to decide in favor or against toolcoupling. When considering small or numerically simple models, the simulation time is most likely to increase when splitting them into several parts (see e.g. [9]). Looking at large or complex models, splitting these into submodels can greatly enhance the speed.

Especially solving systems of equations with significantly different time constants can be greatly improved by decoupling these time constants. While simulation tools could support this internally by employing multi-rate solving techniques, co-simulation enables the user to create a "distributed multi-rate simulation" using tools without a multi-rate solver.

Considering all points mentioned above it becomes clear that the model partitioning is an important part of the model design. Normally aspects like time constants or required computing time have to be inspected. This step can be omitted for this paper, since each 
component shall be simulated separately.

\section{Co-simulation}

For the tool coupling this paper uses the co-simulation environment TISC ${ }^{\circledR}$. This environment is divided in two layers: The Control-Layer and the SimulationLayer (figure 1). To ensure platform independency, TCP-sockets are used for communication between all distributed components.

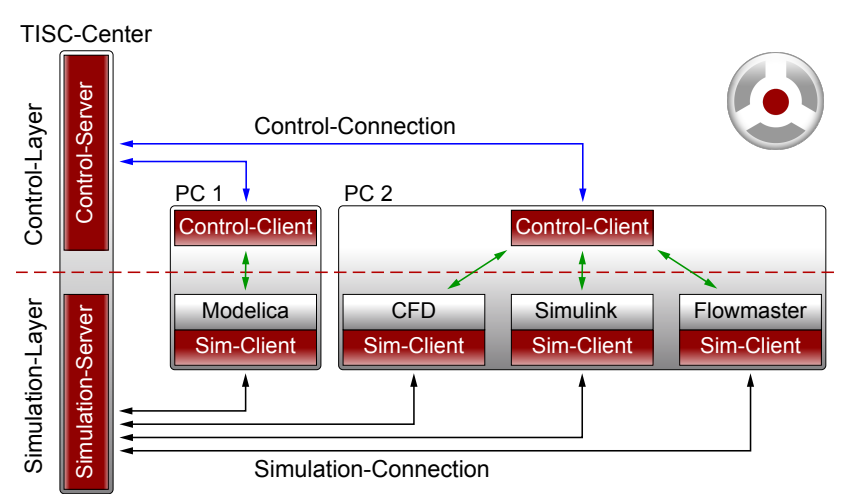

Figure 1: Layer structure of TISC

The Control-Layer consists of the central ControlServer and several Control-Clients - one on each computer participating in the simulation. Through the Control-Server the user can trigger the start of the simulation. The Control-Server sends the appropriate commands to the respective Clients using the ControlConnection. Besides these start commands, also status messages and stop commands can be sent.

The started model instances connect to the Simulation-Server through the Simulation-Client, which has been integrated into each model. The used integration techniques differs depending on the used language and tool. In case of Modelica and Simulink input and output blocks are added to the model, in case of the $1 \mathrm{~d}$ tools Flowmaster and Kuli the information is accessed through interfaces available through COM, other tools (e.g. CFD) require still other techniques.

To make it more convenient for the user to configure, run and evaluate the simulation, the two Servers are united in the TISC-Center.

During the simulation, the Simulation-Server's tasks are the data transfer between and synchronization of the single models. While it is possible to use sequential (or "explicit") synchronization, the parallel (or "implicit") synchronization (see [11]) is used most of the time in TISC. As main advantage of this over the sequential synchronization, the different models are being calculated in parallel. This benefit is amplified with an increasing number of coupled models leading to a major increase of simulation speed for complex systems.

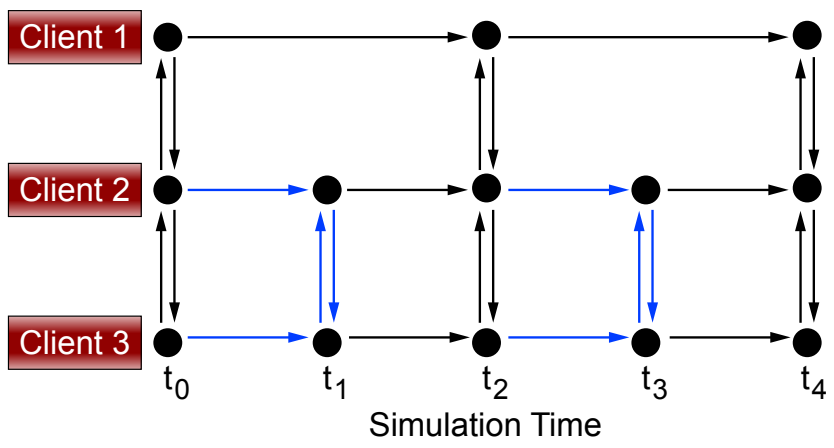

Figure 2: TISC snychronization scheme

The required time for the co-simulation is heavily influenced by the exchange rate between the single models. At synchronization time, every client has to be stopped, the data transmitted and the solvers reinitialized. While the time needed for reinitialization heavily depends on the employed solver, the other delays are directly proportional to the number of synchronization points. As shown in figure 2, the implemented synchronization allows for different time step sizes for the simulation clients. Therefore the overall simulation speed can be improved by increasing the exchange rate for complex systems with relatively large time constants thus reducing the overall number of synchronization events.

At synchronization time the reinitialization of the solvers is being hampered by the value patterns of the variables exchanged through TISC. Since only the value of the variables can be transferred, the variable is a discrete one on the receiving side. The higher the step at synchronization time, the harder it is for the solver to find a consistent solution for the system of equations - it is even possible that the solver fails to find a solution. TISC includes extrapolation and smoothing possibilities on the receiving side to cancel this effect. Figure 3 shows the values of a sine sent through TISC with a period time of $1 s$ and an exchange rate of $0.1 \mathrm{~s}$. Cubic polynomials are used to extrapolate received values to the respective next time step. By using a 5th degree polynomial to switch between the polynomial built from the 4 values before synchronization time and the polynomial built from the last 4 values including the synchronization time, the curve is smoothed resulting in the line shown in figure 3 ("Extrapolated"). The described technique leads to a function which is two times continuously differentiable, hereby helping the solving process. 


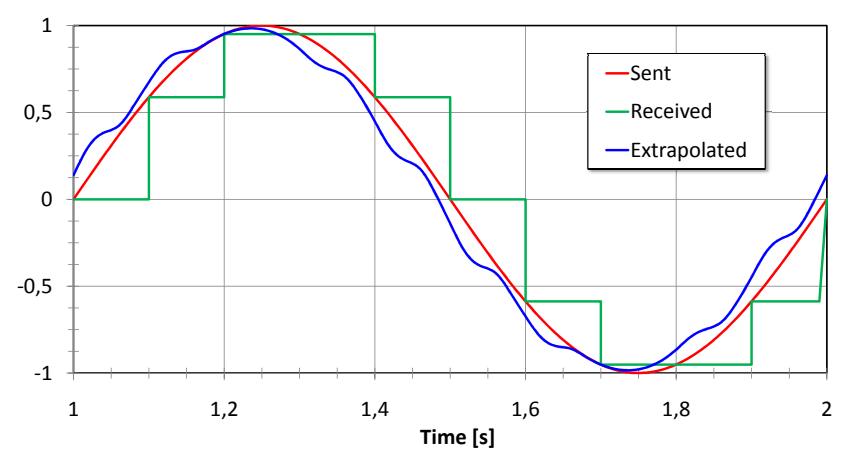

Figure 3: Value pattern with and without extrapolation

\section{Investigated System}

Figure 4 depicts the design of a car's HVAC unit which consists of five components: a compressor, a condenser, a receiver, an expansion device, an evaporator and an internal heat exchanger.

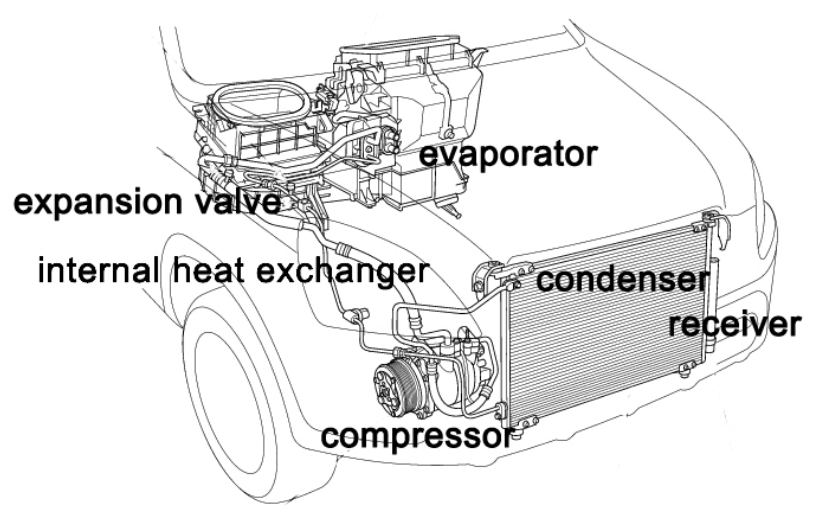

Figure 4: HVAC unit of a car

The HVAC unit uses a circulating refrigerant which enters the compressor and is compressed to a higher pressure, resulting in a higher temperature as well. Afterwards the refrigerant enters the condenser, where it rejects heat to the environment. In the internal heatexchanger the refrigerant is furthermore subcooled by rejectig heat to the low pressure side. Hereafter it is expanded to a lower pressure by an expansion device, e.g. a thermostatic expansion valve. Then the refrigerant flows through the evaporator where it is evaporated. During the process of evaporation the refrigerant absorbs heat from the passenger compartment decreasing its temperature. The absorbed heat is also called cooling capacity, which is a characteristic value of the performance of an HVAC unit. Finally, inside the low pressure side of the internal heat exchanger, the refrigerant is superheated before reentering the compressor.

\subsection{Component modeling}

The R134a vapor compression cycle is modeled using component models from the TIL library. TIL is a component model library for thermodynamic systems developed by the Institute for Thermodynamics (IfT) and the TLK-Thermo GmbH. It allows for steady-state and transient simulation of thermodynamic systems.

Heat transfer and pressure drop correlations for each component model are validated with measurement data from a set of more than 15 different ambient conditions.

The condenser is a flat-tube heat exchanger with four refrigerant flow passes. Each of the four passes is discretized into five control volumes ("cells") representing the manifoldness of flat-tubes of the respective pass, thereby considering different flow cross sectional areas. Since the receiver is integrated into the condensor, the component is also called "CondReceiver".

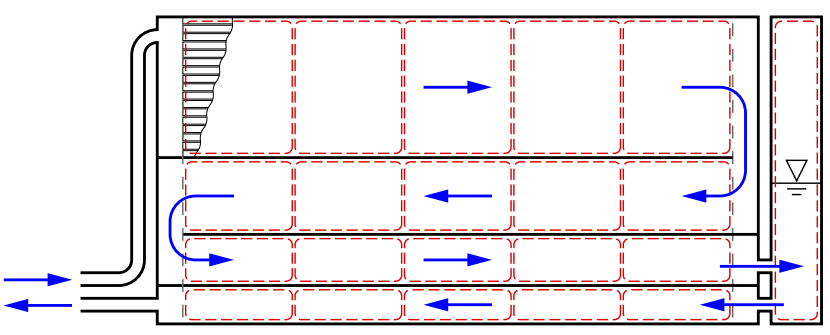

Figure 5: Schematic diagram of CondReceiver

Air-side heat transfer coefficient and pressure drop are modeled using correlations from literature [10] capturing influences of geometry as well as ambient conditions. The refrigerant side heat transfer coefficient is preestimated using a correlation specific for condensation in minichannels [2] but is set constant during the simulation to the preestimated value of $\alpha=$ $4300 \mathrm{~W} / \mathrm{m}^{2} \mathrm{~K}$. Wall heat conduction is modeled onedimensional and perpendicular to both fluids, where characteristic lengths are calculated from the geometric parameters. The integrated receiver is considered as a separator with a characteristic curve accounting for changes in outlet vapor fraction at very low and very high filling levels.

Due to the object oriented approach, the evaporator model is built from the same basic elements as the condenser - with different geometric parameters. The evaporator modeled has a two layer design with three passes per layer. Each of the passes is discretized into five cells. Condensation and evaporation of moisture are taken into account by means of an analogy of heat and mass transfer. The heat transfer and pressure drop correlations are developed anal- 
ogous to those of the condenser. The refrigerant side heat transfer coefficient is also set to a preestimated value $\left(\alpha=4300 \mathrm{~W} / \mathrm{m}^{2} \mathrm{~K}\right)$ during simulation.

The internal heat exchanger is modeled as a tube in tube heat exchanger, using heat transfer correlations from [13]. Each tube is represented by five cells.

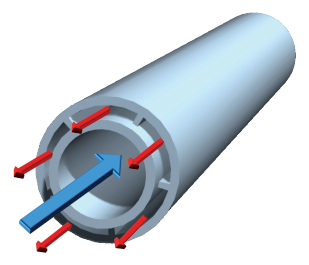

Figure 6: Schematic drawing of internal heat exchanger

The compressor model is mapped using a quasisteady state model based on measurement data for full and partial load as proposed by [3]. Three efficiency functions are used to characterize the compressor efficiencies, namely volumetric efficiency, effective isentropic efficiency and isentropic compressor efficiency.

The thermostatic expansion valve is modeled using Bernoulli's equation for compressible and incompressible flow [4].

\subsection{Model validation}

Even though data validity is usually not considered part of model verification and validation, it is addressed here, as suggested by [12], as measurement data validation sets the baseline for the achievable model accuracy. Therefore only a small proportion of the available measurement data was used for validation purposes.

Simulations in test bench environments have been run for all component models. In these test benches, each component model is provided with mass flow, specific enthalpy and pressure by boundaries at the inlet or the outlet. These boundary conditions are extracted from measurement data for over 15 measurement points. Extreme-condition Tests were run to define the scope of each component model.

Exemplarily the validation results of the condenser model are depicted in figure 7 in terms of measured rejected heat over simulated rejected heat.

The measurement data can be reproduced with a deviation of $\pm 10 \%$ by using empirical, physically motivated correlations without any correction factors. These points cover a range of thermal efficiency of $65 \%$ to $100 \%$.

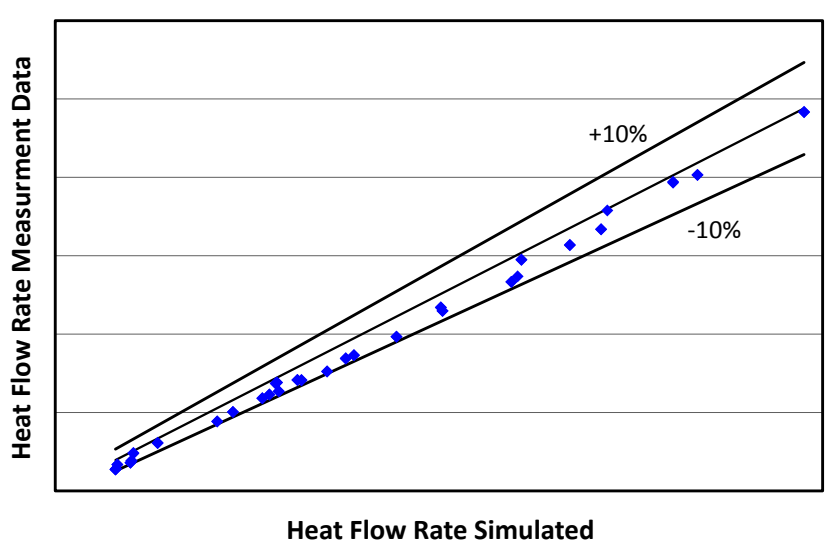

Figure 7: Comparison of measurement data and simulated heat flow rate with validated condenser model

After combining the single models to a closed model of the refrigeration cycle, p,h-diagrams were used as further means of validation. Figure 8 depicts the slight deviations of the simulation results compared to the measurement data stating the validity of the system model.

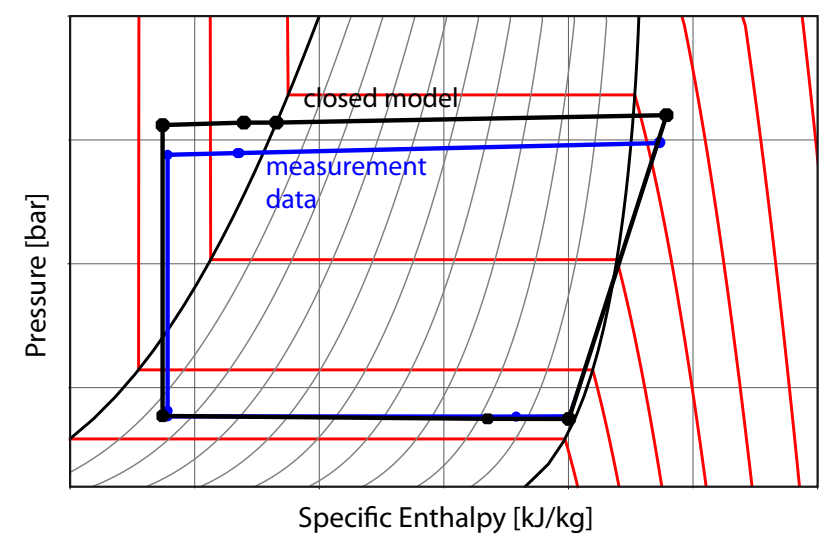

Figure 8: Comparison of measurement data and simulation results of a steady state condition

\section{Simulation setup}

In order to determine the effects of tool coupling on the simulation of the closed refrigeration cycle, the single validated models presented in section 3 are connected in two different ways:

\section{Closed model in Modelica \\ 2. Co-simulation of separate models}

When splitting a model into submodels in preparation of a co-simulation, several possibilities exist. Various considerations may influence the partitioning. 
When different tools are to be employed - e.g. coupling a 3d-model of a heat exchanger with 1d-models of the remaining components - the cutting points are obvious. Very detailed component models can be separated in order to use more processors and memory. Models with considerably different time constants can be decoupled creating a sort of distributed multirate method increasing the overall simulation speed $[6,7]$.

The system presented in this paper is composed of detailed models (especially the heat exchangers) with many interconnections. Since the intention was to reuse the models of section 3 without modifications, they were in the first step included in the co-simulation as independent systems. Since the small time constant of the internal heat exchanger induces a tight coupling of the two pressure levels, the complete system is prone for oscillation of the thermodynamic state variables. In addition, the complex heat transfer and pressure drop correlations impede the simulation. In a second step therefore the internal heat exchanger was split into two parts in order to get closed models of the two pressure levels which correspond to one pressure state variable each in TIL [8]. Figure 9 shows the structure of the closed refrigeration cycle, the colored areas corresponding to the four coupled models Compressor (green), CondReceiver and high pressure side of the Internal Heat Exchanger (blue), Valve (brown) and Evaporator and low pressure side of the Internal Heat Exchanger (red).

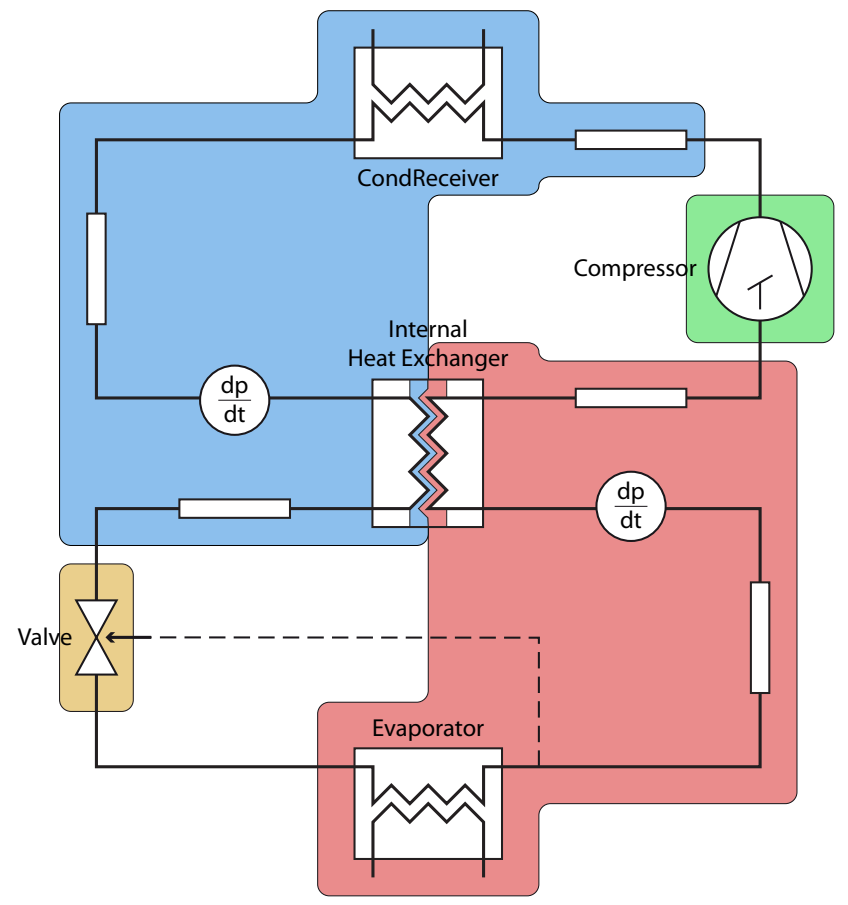

Figure 9: Structure of simulated cycle
As figure 10 shows, the internal heat exchanger is represented by tubes with heat ports. The heat flow rate is read on the high pressure side and imposed on the low pressure side. The temperature is treated the same way in the opposite direction. Resistors are added to each side to increase the time constant of the subsystems. Furthermore a capacitor is used to create an artificial temperature state. While the effect of these three elements on the accuracy of the steadystate simulation result is negligibly small, they allow for a larger larger exchange rate in the co-simulation.

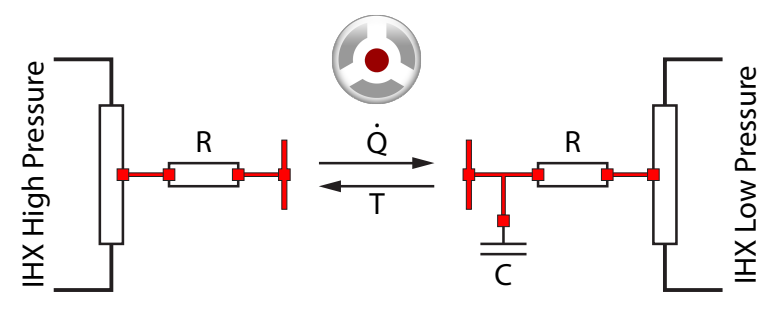

Figure 10: Splitting of Internal Heat Exchanger for cosimulation

To be able to compare the closed model with the cosimulation, the same component models with identical sets of parameters are used. The correlations for heat transfer and pressure drop are switched from constant values during initialization to geometrically and physically based correlations at different instances in time during the simulation. This procedure has shown to be necessary for the closed Modelica model to achieve a robust initialization. Although the co-simulation models can be started using the complex correlations from the very beginning, the same settings were used for the correlations for better comparability of the results.

\section{Effects of co-simulation on steady- state simulation}

The main differences for the user are development time and the time needed for the simulation. In figure 11 the simulation time for different simulations is presented. The switching of correlations is deductable from the pattern of the closed model (green line). The red and the blue line represent coupled simulations varying only in the employed solver (the time needed for the simulation using the dassl solver is about 5800 seconds wall clock time for 60 seconds simulation time).

Comparing the co-simulation with the closed model, a gain in speed is achieved during initialization. As the curves of the coupled simulations highlight, the co-simulation is able to initialize a lot faster 


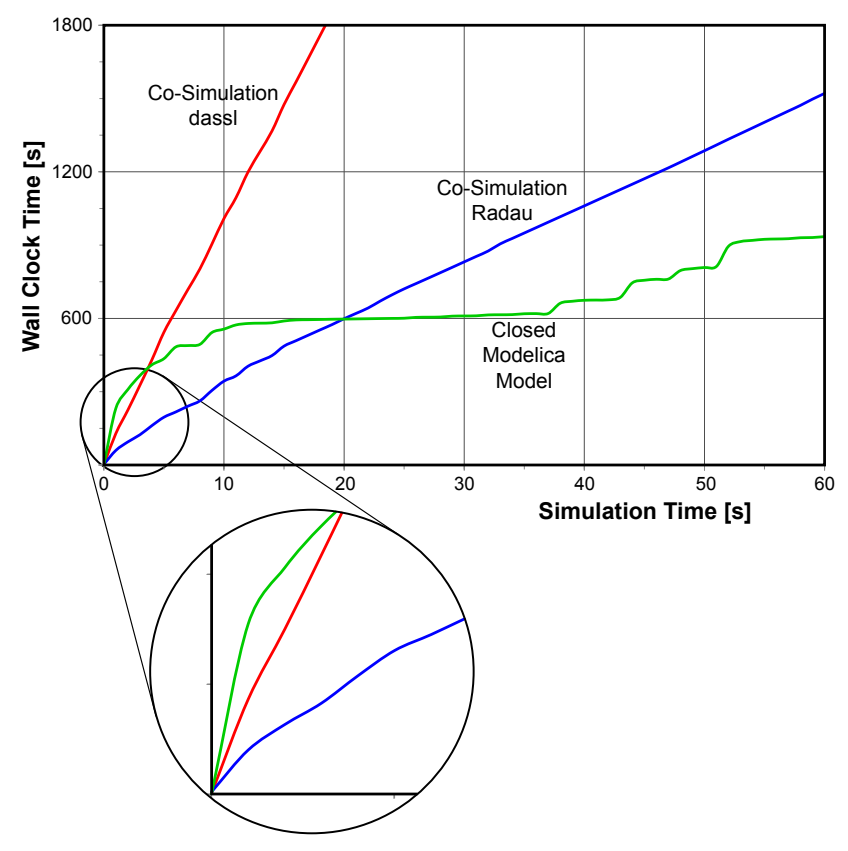

Figure 11: Simulation time of different simulations with constant boundary conditions

- regardless of the employed solver. Experience also shows that the co-simulation is far less vulnerable to ill-configured start and initial values. For this reason the development time needed for reaching a well behaving simulation is much lower when employing cosimulation.

As figure 12 highlights, the result of the cosimulation is consistent with the one from the closed model. Analogous to section 3.2, the co-simulation of the refrigeration cycle is considered as validated.

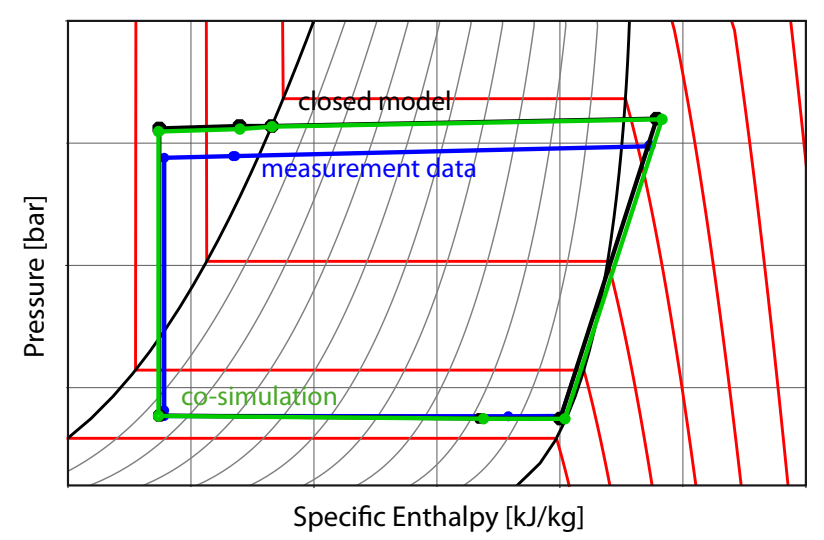

Figure 12: p,h-diagram of a simulated steady state condition

The data exchange rate not only has effect on the required simulation time as stated in section 2 , it also heavily influences the robustness of the co-simulation - the smaller the exchange rate the more robust the simulation. The drawback of a small exchange rate is the decrease in simulation speed. Since the numerical solver has to be stopped at a specific point in time, not only the time event is generated, the solver also needs to be reinitialized which requires a significant amount of time [1]. As figure 11 shows, the chosen solver can also greatly influence the simulation speed. The size of the data exchange rate is limited by the time constants of the system's components. The simulation shown in figures 11 and 12 were conducted with an exchange rate of 0.1 seconds, which roughly equals the lowest time constant in the system.

\section{Effects of co-simulation on tran- sient simulation}

The advantage of the co-simulation reacting friendly to ill-configured starting conditions can also be observed during transient simulations with highly dynamic boundary conditions. As an example a simulation using the NEDC (see figure 13) was conducted.

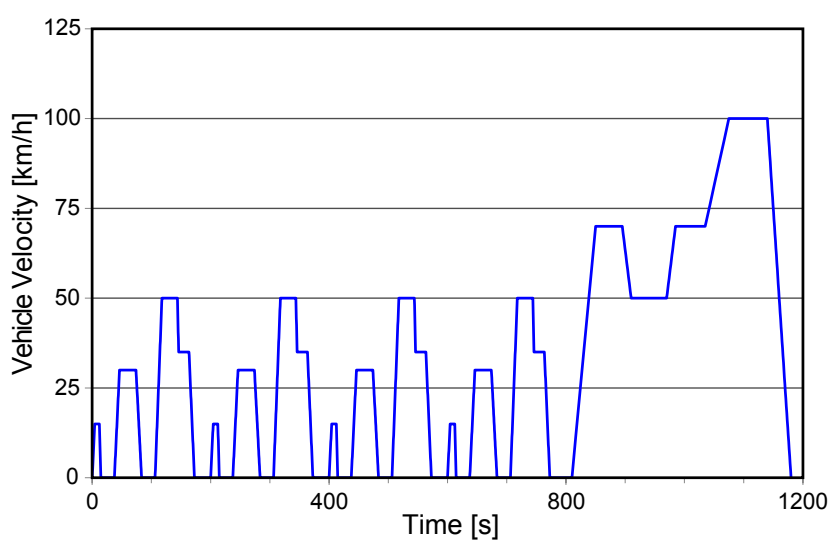

Figure 13: New European Driving Cycle (NEDC)

While the time constants can be easily determined under constant boundary conditions, close attention has to be paid in a changing environment. During periods with highly dynamic boundaries the smallest time constant was as low as 0.02 seconds. Therefore the data exchange rate had to be adjusted to these changing conditions since a slightly too large rate is immediately inducing instabilities.

\section{Conclusion and Outlook}

Modelica models for the different components of a car's HVAC unit have been modeled and validated in separate test benches. It could be shown, that a closed model of a refrigeration cycle employing the validated 
components as well as a co-simulation coupling separate simulation instances of the same components can be considered validated.

To reach a robust initialization for the closed model, heat transfer and pressure correlations were set to start with constant values, switching to physically motivated equations at different instances in time during the simulation. Furthermore, adjustments to start and initial values had to be made. The co-simulation required no simplified correlations and also initialized robustly with ill-configured start and initial values. Therefore the co-simulation can play it's trump cards when changing single component models of an existing system or when building a completely new system model. In addition, also the simulation of different constant and dynamic boundary conditions is simplified. In all cases, the start and initial values hardly ever need to be changed.

Different techniques can be utilized to further stabilize the solution process during a co-simulation. Decoupling of tight dependencies was presented by splitting the internal heat exchanger thus separating the two pressure state variables of the refrigeration cycle. Extrapolation and smoothing can be applied to avoid steps in the course of received values simplifying the reinitialization of the numerical solver. An investigation targeting the optimal extrapolation order has not been conducted for this paper but is interesting for future work since the critical data exchange rate is smaller at higher orders of extrapolation (see [7]). Even if not using extrapolation, smoothing the steps still helps the solver.

Close attention has to be paid to the data exchange rate within the co-simulation since it must never exceed the smallest time constant. While this time constant can easily be determined in simulations with constant boundary conditions, it is more difficult but not less important with dynamic boundaries like driving cycles. An automatic adjustment of the exchange rate is subject of future work.

\section{References}

[1] M. Arnold, Simulation Algorithms in Vehicle System Dynamics, Martin-Luther-Universität Halle-Wittenberg, 2004.

[2] T. M. Bandhauer, Measurement and Modeling of Condensation Heat Transfer Coefficients in Circular Microchannels, In: Transactions of the ASME, Vol. 128, 2006.
[3] S. Försterling, Vergleichende Untersuchung von CO2-Verdichtern in Hinblick auf den Einsatz in mobilen Anwendungen, TU Braunschweig, PhD-Thesis, 2004.

[4] D. W. Green, Perry's chemical engineers' handbook, The McGraw Hill Companies, ISBN 9780-07-142294-9, 2007.

[5] R. Kossel et al., Simulation of Complex Systems using Modelica and Tool Coupling. In: Proceedings of the 5th International Modelica Conference 2006, Vienna, Austria, Modelica Association, 4-5 September 2006.

[6] R. Kossel et al., Einsatz hybrider Simulationstechnik für die Bewertung mobiler Heiz- und Kühlkonzepte. In: Wärmemanagement des Kraftfahrzeugs VI, Berlin, Germany, Haus der Technik, June 2008.

[7] R. Kübler, Modulare Modellierung und Simulation mechatronischer Systeme, Universität Stuttgart, PhD-thesis, 2000.

[8] N. Lemke, Untersuchung zweistufiger Flüssigkeitskühler mit dem Kältemittel $\mathrm{CO} 2$, TU Braunschweig, PhD-thesis, 2004.

[9] K. Nyström and P. Fritzson, Parallel Simulation with Transmission Lines in Modelica. In: Proceedings of the 5th International Modelica Conference 2006, Vienna, Austria, Modelica Association, 4-5 September 2006.

[10] Y.-G. Park et al., Air-Side Heat Transfer and Friction Correlations for Flat-Tube Louver-Fin Heat Exchngers, In: Journal of Heat Transfer, February 2009, vol. 131.

[11] W. Puntigam et al., Transient Co-Simulation of Comprehensive Vehicle Models by Time Dependent Coupling. In: SAE 2006 Transactions Journal of Passenger Cars: Mechanical Systems, ISBN 978-0-7680-1838-7, pages 1516 - 1525.

[12] Robert G. Sargent, A tutorial on validation and verification of simulation models, In: Proceedings of the 1988 Winter Simulation Conference, San Diego, USA, 12-14 December 1988.

[13] John R. Thome, Engineering Data Book III, 2004. 
Proceedings 7th Modelica Conference, Como, Italy, Sep. 20-22, 2009

[14] J. R. Thome, Heat transfer model for evaporation in microchennels Part 1: presentation of the model, In: International Journal of Heat and Transfer, March 2004, vol. 47, pp. 3375-3385. 